\title{
Cut Elimination in Multifocused Linear Logic
}

\author{
Taus Brock-Nannestad \\ INRIA \& LIX, École Polytechnique \\ taus.brock-nannestad@inria.fr
}

\author{
Nicolas Guenot \\ IT University of Copenhagen \\ ngue@itu.dk
}

\begin{abstract}
We study cut elimination for a multifocused variant of full linear logic in the sequent calculus. The multifocused normal form of proofs yields problems that do not appear in a standard focused system, related to the constraints in grouping rule instances in focusing phases. We show that cut elimination can be performed in a sensible way even though the proof requires some specific lemmas to deal with multifocusing phases, and discuss the difficulties arising with cut elimination when considering normal forms of proofs in linear logic.
\end{abstract}

\section{Focusing and Multifocusing in Linear Logic}

The two most important results in the proof theory of linear logic [6] are the admissibility of the cut rule, and the completeness of the focused normal form of proofs. The notion of focusing was originally developped by Andreoli [1] with the purpose of improving proof search procedures, but recently it has been considered more often as a normal form that can be obtained by reorganising the inference steps of a given proof [10]: permutations can be used to group positive rule instances — and to move negative rule instances down. This viewpoint is particularly useful in the natural extension of focusing to multifocusing [5], where several positive formulas are selected to be decomposed in parallel. This stronger normal form is difficult to use for proof search, since not all positive formulas can be selected in a given sequent: there are complex dependencies. In the multiplicative fragment without units, when the selection of positives is done maximally, proofs are canonical in the sense that they are in bijection with proof-nets [7]. For this reason, investigating the proof theory of multifocused linear logic is necessary to understand the notion of canonicity in sequent calculi, and possibly design normal forms of proofs that could be used as proof-nets, while retaining the usual syntax based on trees of rule instances.

Our purpose here is to study the interaction of cut elimination with multifocusing. We consider the sequent calculus LLM shown in Figure 1, which is equivalent to the one found in [11]. It relies on a polarised syntax [9] where shifts mark borders between positive and negative connectives, as follows:

$$
\begin{array}{c|c|c|c|c|c|c}
P, Q::=a & 1 & P \otimes Q & 0 & P \oplus Q & ! N & \downarrow N \\
N, M::=\bar{a} & \perp & N \& M & \top & N \& M & ? P & \uparrow P
\end{array}
$$

and we write $P^{\perp}$ for the usual duality operation of linear logic. Moreover, this system uses sequents of the shapes $\vdash \Omega: \Gamma$ and $\vDash \Omega: \Psi$ for inversion and focusing phases respectively, where the names used for multisets denote various syntactic categories:

$$
\Gamma, \Delta::=\cdot|\Gamma, N \quad \Psi, \Xi::=\Gamma| \Psi,[P] \quad \Theta, \Omega::=\cdot \mid \Theta, P
$$

and the multiset $\Omega$ of positives on the left of the sequent is the persistent context, corresponding to a multiset of formulas that can be duplicated. Finally, the decision rule $\uparrow$ uses the special syntax $\Omega^{\vec{n}}$ to denote a multiset made of arbitrary numbers of copies of formulas in $\Omega$ - since this rule needs to allow choosing formulas from $\Omega$ to focus on, and possibly several copies of the same formula.

This work is licensed under the

\section{Creative Commons Attribution License.}




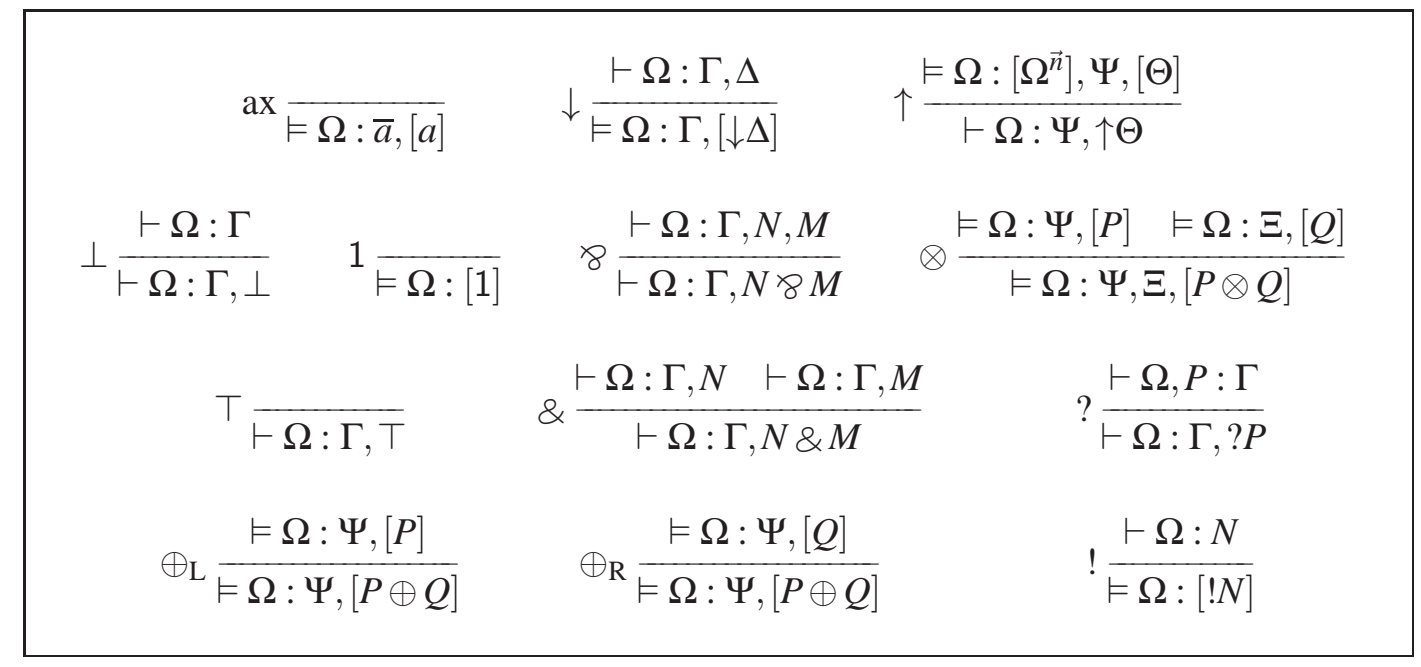

Figure 1: Multifocused sequent calculus LLM for linear logic

The most important rules for multifocusing are the $\uparrow$ and $\downarrow$ rules, that start and end the focusing phases, respectively. These two rules act on multisets of formulas rather than on single formulas, and in particular it is important that all foci are blurred at once in the $\downarrow$ rule, so that the different phases are clearly separated. They come with side conditions:

- in the $\uparrow$ rule, either $\Omega^{\vec{n}}$ or $\Theta$ must be non-empty, and

- in the $\downarrow$ rule, the multiset $\Delta$ must be non-empty.

The LLM system is slightly different from other presentations [5, 11] in that it does not enforce maximal inversion of negative formulas before a focusing phase can start. From the viewpoint of cut elimination, it makes no difference, and proving the admissibility of cut for the more permissive system implies that cut elimination holds for the corresponding system where inversion is performed maximally. Moreover, we are interested in developing a proof technique for admissibility of cut in multifocused systems that could be used when negative formulas are treated differently.

Example 1. The proof shown below uses multifocusing to treat the formulas $\uparrow(a \otimes \downarrow \bar{b})$ and $\uparrow(c \otimes \downarrow \bar{d})$ in one single focusing phase. Notice that the two lower instances of the $\otimes$ rule could be permuted, but this is irrelevant here since a multifocusing phase should be considered as a "black box". Also, this proof is maximally multifocused, since in both instances of the $\uparrow$ rule, all positive formulas that could be picked are focused.

$$
\begin{aligned}
& \text { ax } \overline{\vDash \cdot: \bar{b},[b]} \quad \text { ax } \overline{\vDash \cdot: \bar{d},[d]} \\
& \uparrow \frac{\vDash \cdot: \bar{b}, \bar{d},[b \otimes d]}{} \\
& \uparrow \overline{\vdash \cdot: \bar{b}, \bar{d}, \uparrow(b \otimes d)}
\end{aligned}
$$

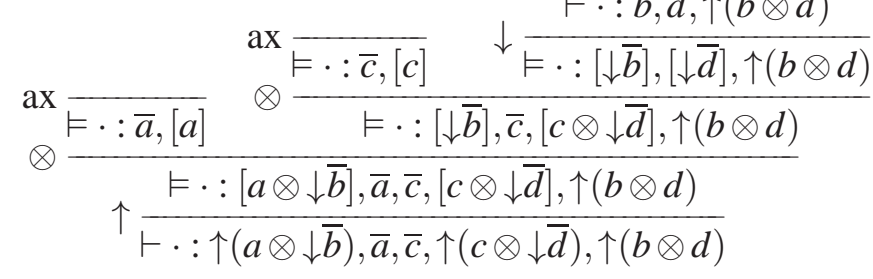




$$
\begin{array}{rc}
\operatorname{cut} \frac{\vDash \Omega: \Psi,[P] \quad \vdash \Omega: \Gamma, P^{\perp}}{\vDash \Omega: \Psi, \Gamma} & \text { fcut } \frac{\vDash \Omega: \Psi,[P] \quad \vDash \Omega: \Xi, P^{\perp}}{\vDash \Omega: \Psi, \Xi} \\
\operatorname{cut} ! \frac{\vdash \Omega: P^{\perp} \quad \vdash \Omega, P: \Gamma}{\vdash \Omega: \Gamma} & \text { fcut! } \frac{\vdash \Omega: P^{\perp} \quad \vDash \Omega, P: \Psi}{\vDash \Omega: \Psi}
\end{array}
$$

Figure 2: Cut rules for LLM

Notice finally that the LLM system is obviously sound with respect to linear logic, in the sense that all focusing annotations can be erased to turn any proof of LLM into a valid proof of a dyadic presentation of linear logic. It is also complete, since it is a generalisation of the usual singly-focused system, just as the other multifocused systems [5, 11].

\section{Cut Elimination with Multifocusing}

Because our system has two different kinds of sequents and two contexts, we get four different cut rules, shown in Figure 2. Note that in the linear cut rules, the first premise must always be a focused sequent, as the cut formula in this premise is positive, and hence must appear inside a focus. Conversely, for cuts acting on the persistent context, the first premise must be an unfocused sequent.

A standard way of proving admissibility of the cut rule is to proceed by lexicographical induction on the structure of the cut formula and the two input derivations. The cases of that proof fall into various categories depending on whether the cut formula is being decomposed (in the so-called principal cases) or whether it simply moves the cut further up in the proof (in the so-called commutative cases). Thus, in the singly-focused system, you might see the following cut:

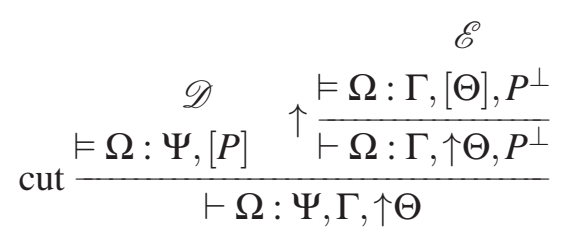

And reduce it as follows:

$$
\begin{aligned}
& \vDash \Omega: \Psi, \Gamma,[\Theta] \\
& \vdash \Omega: \Psi, \Gamma, \uparrow \Theta
\end{aligned}
$$

by fcut on $P, \mathscr{D}, \mathscr{E}$.

by $\uparrow$.

In the singly-focused system, $\Psi$ cannot contain any focus 1 , hence the resulting sequent after the cut contains only the foci in $\Theta$, and thus the $\uparrow$ rule can be applied. In the multifocused system, this is no longer the case $-\Psi$ may contain several foci, and thus we cannot be sure that the side condition on the $\uparrow$ rule is satisfied.

There are various ways one might try to fix the above problem. One way would be to change the cut rule itself to make the conclusion more permissive. For instance, if we define the following neutralising operation:

$$
\langle\Gamma\rangle=\Gamma \quad\langle\Psi,[P]\rangle=\langle\Psi\rangle, \uparrow P
$$

\footnotetext{
${ }^{1}$ Additionally, $\Theta$ must consist of a single formula, but this is not important.
} 
we can restate the cut rules as

$$
\operatorname{cut} \frac{\vDash \Omega: \Psi,[P] \quad \vdash \Omega: \Gamma, P^{\perp}}{\vdash \Omega:\langle\Psi, \Gamma\rangle} \quad \text { fcut } \frac{\vDash \Omega: \Psi,[P] \vDash \Omega: \Xi, P^{\perp}}{\vdash \Omega:\langle\Psi, \Xi\rangle}
$$

and the above proof would then go through, as $\langle\Psi, \Gamma,[\Theta]\rangle=\langle\Psi\rangle, \Gamma, \uparrow \Theta=\langle\Psi, \Gamma, \uparrow \Theta\rangle$. The delayed cut above is somewhat weak, however, as it forces a delay even in cases where it is not necessary.

As we will show now, it is not necessary to change the statement of the cut rule to prove it admissible. To circumvent the above problem, we will instead introduce a few lemmas about the structure of the positive phases. First, we introduce the notion of a spent context $\Sigma$ :

$$
\Sigma::=\Gamma \mid \Sigma,[\downarrow N]
$$

Intuitively, a context $\Sigma$ may contain foci, but none of these foci can be active anymore. The main purpose of this definition is to facilitate the proof of the following lemma, which will play an important role in the cut admissibility proof.

Lemma 1 (Multifocused Decomposition). Given a proof of a sequent $\vDash \Omega: \Psi,[P]$ there exists:

1. a proof of the sequent $\vDash \Omega: \Sigma,[P]$, for some suitable $\Sigma$, and

2. for any $\Delta$, an open derivation from $\vDash \Omega: \Sigma, \Delta$ to $\vDash \Omega: \Psi, \Delta$.

The combined height of these two derivations is exactly the height of the input derivation.

Proof. Let $\mathscr{D}$ be the given derivation of $\vDash \Omega: \Psi,[P]$. We proceed by induction on the structure of $\mathscr{D}$. When we apply the induction hypothesis in the remainder of this proof, we will refer to the first derivation as $\mathscr{D}^{\prime}$, and the open derivation instantiated with $\Delta$ as $\mathscr{E}_{\Delta}^{\prime}$.

- Case $\Psi=\Sigma$ : Immediate. This case also covers the cases where $\mathscr{D}$ ends in the 1 , ax or $\downarrow$ rules.

- Case $\otimes, P=Q \otimes R$ principal:

$$
\begin{aligned}
& \mathscr{D}_{1} \quad \mathscr{D}_{2} \\
& \otimes \frac{\vDash \Omega: \Psi_{1},[Q] \quad \vDash \Omega: \Psi_{2},[R]}{\vDash \Omega: \Psi_{1}, \Psi_{2},[Q \otimes R]}
\end{aligned}
$$

We construct $\mathscr{D}^{\prime}$ as follows:

$$
\begin{aligned}
\mathscr{D}_{1}^{\prime}:: & \vDash \Omega: \Sigma_{1},[Q] \\
\mathscr{D}_{2}^{\prime}:: & \vDash \Omega: \Sigma_{2},[R] \\
& \vDash \Omega: \Sigma_{1}, \Sigma_{2},[Q \otimes R]
\end{aligned}
$$

by the induction hypothesis on $\mathscr{D}_{1}$. by the induction hypothesis on $\mathscr{D}_{2}$. by $\otimes$ on $\mathscr{D}_{1}^{\prime}, \mathscr{D}_{2}^{\prime}$.

We construct $\mathscr{E}_{\Delta}^{\prime}$ as follows:

$$
\begin{aligned}
& \vDash \Omega: \Sigma_{1}, \Sigma_{2}, \Delta \\
& \vDash \Omega: \Sigma_{1}, \Psi_{2}, \Delta \\
& \vDash \Omega: \Psi_{1}, \Psi_{2}, \Delta
\end{aligned}
$$

by assumption. by $\mathscr{E}_{2 \Delta, \Sigma_{1}}^{\prime}$. by $\mathscr{E}_{1 \Delta, \Psi_{2}}^{\prime}$.

- Case $\otimes, P$ not principal:

$$
\otimes \frac{\vDash \Omega: \Psi_{1},[Q],[P]}{\mathscr{D}_{1}} \quad \begin{gathered}
\mathscr{D}_{2} \\
\vDash \Omega: \Psi_{1}, \Psi_{2},[Q \otimes R],[P]
\end{gathered}
$$

We construct $\mathscr{D}^{\prime}$ as follows:

$$
\vDash \Omega: \Sigma,[P]
$$

by the induction hypothesis on $\mathscr{D}_{1}$. 
We construct $\mathscr{E}_{\Delta}^{\prime}$ as follows:

$$
\begin{aligned}
& \vDash \Omega: \Sigma, \Delta \\
\mathscr{D}_{1}^{\prime \prime}:: & \vDash \Omega: \Psi_{1},[Q], \Delta \\
& \vDash \Omega: \Psi_{1}, \Psi_{2},[Q \otimes R], \Delta
\end{aligned}
$$

by assumption.

by $\mathscr{E}_{1 \Delta}^{\prime}$.

by $\otimes$ on $\mathscr{D}_{1}^{\prime \prime}$ and $\mathscr{D}_{2}$.

The remaining cases are similar.

In some cases, we will make use of the following observation about sequents of the form $\vDash \Omega: \Sigma$. First we will define a lowering operation that inverts spent foci:

$$
\lfloor\Gamma\rfloor=\Gamma \quad\lfloor\Sigma,[\downarrow N]\rfloor=\lfloor\Sigma\rfloor, N
$$

With the above definition, the following lemma is an easy consequence:

Lemma 2 (Lowering spent foci). The following rule is admissible:

$$
\downarrow^{-1} \frac{\vDash \Omega: \Sigma,[P]}{\vDash \Omega:[\Sigma\rfloor,[P]}
$$

Furthermore, it is strongly admissible, in the sense that applying the rule does not change the shape of the resulting derivation.

Proof. By induction on the given derivation of $\vDash \Omega: \Sigma,[P]$. The crucial observation is the fact that since $\Sigma$ only contains spent foci, the only active formula is $P$. The base case is when $P=\downarrow N$, and in this case the only rule that could produce the sequent $\vDash \Omega: \Sigma,[\downarrow N]$ is the $\downarrow$ rule applied to the sequent $\vdash \Omega:\lfloor\Sigma\rfloor, N$. By applying the $\downarrow$ rule again, but this time only to the formula $N$, we get the desired conclusion.

With the above lemmas and rules, we can now tackle the admissibility of the cut rules:

Theorem 3 (Admissibility of cut). The rules in Figure2 are admissible in LLM.

Proof. By lexicographic induction on the cut formula and the derivations. We show here a representative selections of cases, in particular some of the principal and boundary cases of the linear cuts. Because we have the decomposition lemma, we can use it on the first subderivation of each rule. It is therefore sufficient to prove the admissibility of the following "spent cut" rules:

$$
\text { scut } \frac{\vDash \Omega: \Sigma,[P] \quad \vdash \Omega: \Gamma, P^{\perp}}{\vDash \Omega: \Sigma, \Gamma} \quad \text { fscut } \frac{\vDash \Omega: \Sigma,[P] \quad \vDash \Omega: \Psi_{2}, P^{\perp}}{\vDash \Omega: \Sigma, \Psi_{2}}
$$

By the following transformation:

$$
\begin{aligned}
& \mathscr{D}^{\prime} \quad \mathscr{D}^{\prime} \quad \mathscr{E} \\
& \vDash \Omega: \Sigma,[P] \\
& \mathscr{E}_{[P]}^{\prime} \| \quad \mathscr{E} \\
& \operatorname{cut} \frac{\vDash \Omega: \Psi,[P] \quad \vdash \Omega: \Gamma, P^{\perp}}{\vDash \Omega: \Psi, \Gamma} \\
& \begin{array}{c}
\operatorname{scut} \frac{\vDash \Omega: \Sigma,[P] \quad \vdash \Omega: \Gamma, P^{\perp}}{\vDash \Omega: \Sigma, \Gamma} \\
\mathscr{E}_{\Gamma}^{\prime} \| \\
\vDash \Omega: \Psi, \Gamma
\end{array}
\end{aligned}
$$


In fact, we can always expand the scut rule as follows:

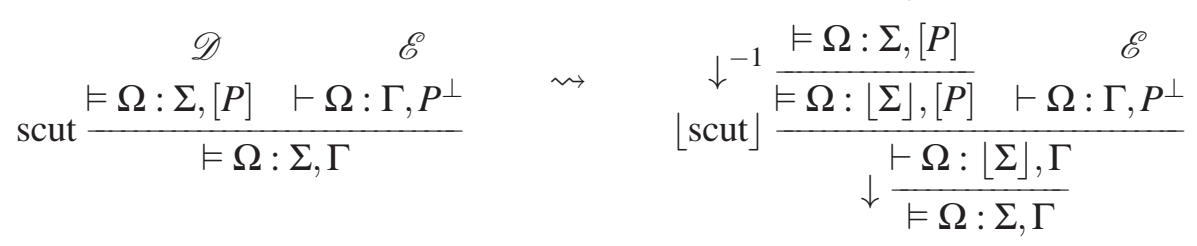

This is justified because the $\downarrow^{-1}$ rule is strongly admissible. It is thus sufficient to show admissibility of the $\lfloor$ scut $\rfloor$ rule. For the principal cuts we reason as follows:

$$
\begin{aligned}
& \begin{array}{lll}
\mathscr{D}_{1} & \mathscr{D}_{2} & \mathscr{E}
\end{array} \\
& \otimes \frac{\vDash \Omega:\left\lfloor\Sigma_{1}\right\rfloor,[P] \quad \vDash \Omega:\left\lfloor\Sigma_{2}\right\rfloor,[Q]}{\models \Omega:\left\lfloor\Sigma_{1}\right\rfloor,\left\lfloor\Sigma_{2}\right\rfloor,[P \otimes Q]} \quad 8 \frac{\vdash \Omega: \Gamma, P^{\perp}, Q^{\perp}}{\vdash \Omega: \Gamma, P^{\perp} \ngtr Q^{\perp}} \\
& \mathscr{E}^{\prime}:: \vdash \Omega:\left\lfloor\Sigma_{1}\right\rfloor, \Gamma, Q^{\perp} \\
& \vdash \Omega:\left\lfloor\Sigma_{1}\right\rfloor,\left\lfloor\Sigma_{2}\right\rfloor, \Gamma \\
& \mathscr{D} \quad \mathscr{E}
\end{aligned}
$$

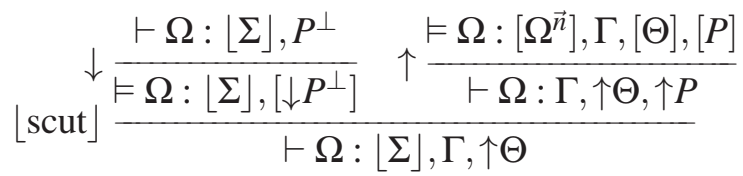

$$
\begin{aligned}
& \vDash \Omega:\lfloor\Sigma\rfloor,\left[\Omega^{\vec{n}}\right], \Gamma,[\Theta] \\
& \vdash \Omega:\lfloor\Sigma\rfloor, \Gamma, \uparrow \Theta
\end{aligned}
$$

Note that we here appeal to the fully general cut rule rather than a more specific rule. In doing so, we implicitly apply the decomposition highlighted at the beginning of this proof.

The commutative cuts are dispensed with in a similar manner. We show here a few instances:

$$
\begin{aligned}
& \mathscr{E} \\
& \lfloor\text { scut }\rfloor \frac{\mathscr{\not}:\lfloor\Sigma\rfloor,[P] \quad \& \frac{\vdash \Omega: \Gamma, P^{\perp}, N, M}{\vdash \Omega: \Gamma, P^{\perp}, N 8 M}}{\vdash \Omega:\lfloor\Sigma\rfloor, \Gamma, N 8 M} \\
& \mathscr{E}^{\prime \prime} \quad:: \vdash \Omega:\lfloor\Sigma\rfloor, \Gamma, N, M \\
& \vdash \Omega:\lfloor\Sigma\rfloor, \Gamma, N \ngtr M \\
& \text { by }\lfloor\text { scut }\rfloor \text { on } P, \mathscr{D}, \mathscr{E} \text {. } \\
& \text { by } 8 \text {. } \\
& \text { fcut } \frac{\mathscr{D} \quad \begin{array}{c}
\mathscr{E} \\
\models \Omega: \Psi,[P]
\end{array} \quad \oplus_{\mathrm{L}} \frac{\vdash \Omega: \Phi,[Q], P^{\perp},}{\vDash \Omega: \Phi,[Q \oplus R], P^{\perp}}}{\vDash \Omega: \Psi, \Phi}
\end{aligned}
$$$$
\mathscr{E}^{\prime} \quad:: \vDash \Omega: \Psi, \Phi,[Q]
$$$$
\vDash \Omega: \Psi, \Phi,[Q \oplus R]
$$

by fcut on $P, \mathscr{D}, \mathscr{E}$.

by $\oplus_{\mathrm{L}}$. 
When the second derivation is at the boundary of a focusing phase, we have restrictions on the shape of the context. In this case, the fact that we can restrict the shape of the context of the first premise of the cut rule becomes crucial.

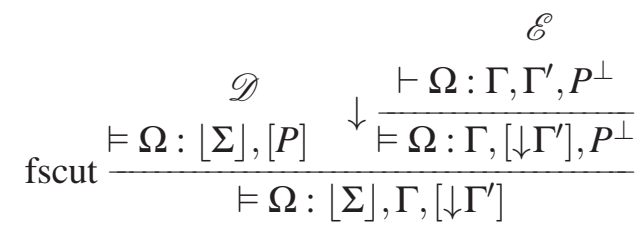

$\vdash \Omega:\lfloor\Sigma\rfloor, \Gamma, \Gamma^{\prime}$

$\vDash \Omega:\lfloor\Sigma\rfloor, \Gamma,\left[\downarrow \Gamma^{\prime}\right]$

$$
\lfloor\text { scut }\rfloor \frac{\mathscr{D} \quad \stackrel{\vDash \Omega \Omega:\left[\Omega^{\vec{n}}\right], \Gamma,[\Theta], P^{\perp}}{\vdash \Omega: \Gamma, \uparrow \Theta, P^{\perp}}}{\vdash \Omega:\lfloor\Sigma\rfloor, \Gamma, \uparrow \Theta}
$$

$\vDash \Omega:[\Sigma\rfloor,\left[\Omega^{\vec{n}}\right], \Gamma,[\Theta]$

$\vdash \Omega:\lfloor\Sigma\rfloor, \Gamma, \uparrow \Theta$ by $\lfloor$ scut $\rfloor$ on $P, \mathscr{D}, \mathscr{E}$.

by $\downarrow$.

by fscut on $P, \mathscr{D}, \mathscr{E}$.

by $\uparrow$.

\section{Further Restrictions on Multifocused Systems}

As we have seen, it is possible to prove cut elimination in a sensible, internal way in the multifocused sequent calculus LLM. However, further restrictions on proofs, designed to obtain stronger normal forms, could yield problems. The obvious restriction to consider here is the one that enforces the maximality of multifocusing [5] - note that such a notion of maximality can be observed on proofs but it is difficult to provide a syntax enforcing maximality. But in this setting, we encounter a problem with the following configuration, for example:

$$
\operatorname{cut} \frac{\mathscr{D}}{\vDash: \uparrow(a \otimes \downarrow \bar{b}),[\downarrow \perp],[\downarrow(\bar{a} \otimes \uparrow b)] \quad \vdash \cdot: \uparrow(a \otimes \downarrow \bar{b}), \bar{a}, \bar{c}, \uparrow(c \otimes \downarrow \bar{d}), \uparrow(b \otimes d)}
$$

where the proof $\mathscr{D}$ is a simple variation of identity expansion, and $\mathscr{E}$ is the proof given in Example 1 both of them being maximally multifocused proofs. Now if we want to perform cut elimination in this restricted system, we expect the resulting proof to be maximally multifocused as well. However, this will separate the phase treating $\downarrow \perp$ from the lower focusing phase in $\mathscr{E}$, although these could be merged into a single phase. Therefore, if this situation arises during cut elimination, with some derivation below that concludes in an unfocused sequent, then the result will not be a maximally multifocused proof. In the presentation of [3], there is a proof that certain cut elimination strategies preserve maximality. However, this is done in the higher-order focusing style due to Zeilberger [13], and this only applies to the MALL fragment of linear logic.

At this point, a better understanding of the dynamics of cut elimination in this setting is required, and in particular we need to control the merging of focusing phases, so that no pair of phases is left that could be turned into a single multifocusing phase. A change in the statement of cut admissibility might be required, or possibly a more elaborate proof technique, potentially involving rewritings even 
less "local" than the ones used in the previous section. In particular, one can consider the following (non-deterministic) activating operation dual to the neutralising one:

$$
\frac{\Psi \uparrow \Xi}{\Psi \cdot} \quad \frac{\Psi \uparrow \Xi}{\Psi, N \uparrow \Xi, N} \quad \frac{\Psi \uparrow \Xi}{\Psi, \uparrow P \uparrow \Xi,[P]} \quad \frac{\Psi,[P] \uparrow \Xi,[P]}{4.1 P]}
$$

and the following kind of cut, where we have $\Psi, \Gamma \uparrow \Xi$ :

$$
\text { acut } \frac{\vDash \Omega: \Psi,[P] \quad \vdash \Omega: \Gamma, P^{\perp}}{\vDash \Omega: \Xi}
$$

so that the conclusion can have more foci than either of the premises. Such a cut would potentially allow the cut shown in (1) to reduce properly, since it offers the possibility to have the multifocused sequent $\vDash \cdot:[a \otimes \downarrow \bar{b}],[\downarrow \perp], \bar{a}, \bar{c},[c \otimes \downarrow \bar{d}], \uparrow(b \otimes d)$ as a conclusion, for which a maximally multifocused proof exists - yielding a maximally multifocused proof of the corresponding unfocused sequent. It suggests that internal cut elimination might be possible in this setting, for an adequate cut.

Alternatively, one could allow foci to be present during the inversion phase, by replacing the $\uparrow$ rule with the following three rules:

$$
\text { focus } \frac{\vDash \Omega: \Psi}{\vdash \Omega: \Psi} \quad \text { copy } \frac{\vdash \Omega, P: \Psi,[P]}{\vdash \Omega, P: \Psi} \quad \uparrow \frac{\vdash \Omega: \Psi,[P]}{\vdash \Omega: \Psi, \uparrow P}
$$

With these rules, it becomes possible to select formulas for focusing one at a time during the inversion phase. This is a somewhat radical step away from the usual presentations of focusing, and so care must be taken to ensure that the resulting system is still well-behaved. With these new rules, the cut rule can now be written as follows:

$$
\operatorname{cut} \frac{\vDash \Omega: \Psi,[P] \quad \vdash \Omega: \Phi, P^{\perp}}{\vdash \Omega: \Psi, \Phi}
$$

Note the two differences in comparison to the rules presented previously. First of all, the context in the second premise can now contain additional foci, and is thus of the form $\Phi$ rather than $\Gamma$. Secondly, the conclusion is in the judgment corresponding to the inversion phase. With these changes, it is no longer necessary to have a concept of spent cuts, and the proof is greatly simplified.

It would be tempting to adopt the following symmetric rules instead of the $\downarrow$ rule:

$$
\operatorname{blur} \frac{\vdash \Omega: \Gamma}{\vDash \Omega: \Gamma} \quad \downarrow \frac{\vDash \Omega: \Psi, N}{\vDash \Omega: \Psi,[\downarrow N]}
$$

Observe that in the context in the blur rule, no foci can be present. This is to prevent foci from "bleeding through" from one phase to the next. Here, however, caution must be exercised, as exchanging the above rules yields a system that fails to enjoy the cut elimination property.

Finally, another interesting restriction of the multifocused system could be defined by controlling inversion phases on negatives in a finer way: instead of maximally decomposing negatives, deal only with the ones that will be "needed" within the next multifocusing phase. Fortunately, the inversion of negatives is not critical in the cut elimination proof, so that such a system would have a strong notion of bipole and yet use the techniques used in the previous section for cut elimination. 


\section{Conclusion and Future Work}

We presented here a proof of cut elimination for a multifocused sequent calculus for linear logic, crucially relying on a decomposition lemma that expresses the fact that parallel phases can be permuted with one another inside a multifocused phase. This kind of order irrelevance is the essence of focusing and, at the "higher" level of focusing phases, this is also the essence of multifocusing: we believe such a lemma can be a useful tool when proving cut elimination for even stronger restrictions of the sequent calculus. Also, operations such as neutralising or activating might prove useful if the precise statement of cut needs to be changed to fit the restrictions of a system.

However, many questions remain open, such as the precise interaction between cut elimination and the organisation of multifocusing phases in a proof, or the definition of a maximally multifocused system equipped with an adequate cut - which could be eliminated entirely inside the system. From a broader perspective, the question of strong normal forms in the sequent calculus or other related systems allowing for permutations is tied to our ability to perform cut elimination in a sensible way under the constraints imposed by multifocusing or even stronger restrictions. For this reason it would be interesting to consider multifocusing in settings such as linear natural deduction [2] or the calculus of structures [4]. From the viewpoint of computation, this means investigating cut elimination as a form of computation in a system where many interleavings of independent steps are abstracted away, as done with proof-nets. It connects the structural approach of standard proof theory to the study of graph-based computational models [8], but this still requires to improve the understanding of normal forms for larger fragments of linear logic.

Finally, extending the notion of focusing raises the question of the elegance of completeness proofs for multifocused systems. Indeed, it seems difficult to prove the focusing result through cut elimination in a simple, natural way [12] if the cut elimination proof itself is complex.

Acknowledgements. This work was partially funded by the Demtech grant number 10-092309 from the Danish Council for Strategic Research.

\section{References}

[1] Jean-Marc Andreoli (1992): Logic programming with focusing proofs in linear logic. Journal of Logic and Computation 2(3), pp. 297-347, doi $10.1093 / \log \mathrm{com} / 2.3 .297$.

[2] Taus Brock-Nannestad \& Carsten Schürmann (2010): Focused Natural Deduction. In C. Fermüller \& A. Voronkov, editors: LPAR-17, LNCS 6397, pp. 157-171, doi 10.1007/978-3-642-16242-8_12.

[3] Kaustuv Chaudhuri (2008): Focusing Strategies in the Sequent Calculus of Synthetic Connectives. In I. Cervesato, H. Veith \& A. Voronkov, editors: LPAR'08, LNCS 5330, pp. 467-481, doi:10.1007/978-3-540-89439-1_33

[4] Kaustuv Chaudhuri, Nicolas Guenot \& Lutz Straßburger (2011): The Focused Calculus of Structures. In M. Bezem, editor: CSL'11, LIPIcs 12, pp. 159-173, doi:10.4230/LIPIcs.CSL.2011.159.

[5] Kaustuv Chaudhuri, Dale Miller \& Alexis Saurin (2008): Canonical Sequent Proofs via Multi-Focusing. In G. Ausiello, J. Karhumäki, G. Mauri \& L. Ong, editors: Fifth IFIP International Conference on Theoretical Computer Science, 273, pp. 383-396, doi:10.1007/978-0-387-09680-326.

[6] Jean-Yves Girard (1987): Linear Logic. Theoretical Computer Science 50, pp. 1-102, doi:10.1016/0304-3975(87)90045-4

[7] Jean-Yves Girard (1996): Proof-nets : the parallel syntax for proof-theory. In A. Ursini \& P. Agliano, editors: Logic and Algebra, M. Dekker, New York. 
[8] Stefano Guerrini, Simone Martini \& Andrea Masini (2001): Proof nets, Garbage, and Computations. Theoretical Computer Science 253(2), pp. 185-237, doi:10.1016/S0304-3975(00)00094-3.

[9] Olivier Laurent (2002): Etude de la polarisation en logique. Thèse de doctorat, Université Aix-Marseille II.

[10] Dale Miller \& Alexis Saurin (2007): From proofs to focused proofs : a modular proof of focalization in Linear Logic. In J. Duparc \& T. A. Henzinger, editors: CSL'07, LNCS 4646, pp. 405-419, doi:10.1007/978-3-540-74915-8_31

[11] Alexis Saurin (2008): Une étude logique du contrôle. Thèse de doctorat, École Polytechnique.

[12] Robert J. Simmons (2014): Structural Focalization. ACM Trans. Comput. Log. 15(3), p. 21, doi: $10.1145 / 2629678$

[13] Noam Zeilberger (2008): On the unity of duality. Annals of Pure and Applied Logic 153(1-3), pp. 66-96, doi:10.1016/j.apal.2008.01.001. 